\title{
Computed Tomography in Orbital Pathology with Clinicopathological Correlation
}

\author{
E.A. Parthasarathy ${ }^{1}$, Einstien. $A^{2}$, Abubacker Sulaiman $\mathrm{F}^{3}$, Alex Daniel Prabhu ${ }^{4}$, Ashraf Ahmed $\mathrm{B}^{5}$, Saravanan $\mathrm{K}^{6}$ \\ ${ }^{1}$ Assistant Professor, ${ }^{2}$ Associate Professor, ${ }^{3}$ Professor and Head of the Department, ${ }^{4}$ Professor, ${ }^{5}$ Associate Professor, ${ }^{6}$ M.D. Final year, \\ Department of Radiology and Imaging Science, Chettinad Hospital and Research Institute, India
}

Corresponding author: Dr. Einstien A, Associate Professor, Department of Radiology and Imaging Science, Chettinad Hospital and Research Institute, Kelambakkam, Kanchipuram- 603103, Tamil Nadu, India.

DOI: $10.21276 /$ ijcmsr.2018.3.2.15

How to cite this article: E.A. Parthasarathy, Einstien A, Abubacker Sulaiman F, Alex Daniel Prabhu, Ashraf Ahmed B, Saravanan K. Computed Tomography in Orbital Pathology with Clinicopathological Correlation. International Journal of Contemporary Medicine Surgery and Radiology. 2018;3(2):B60-B64.

\section{A B S T R A C T}

Introduction: Computed Tomography (CT) is the first-line modality for radiologic evaluation of the orbit in the acute setting. Aim of our study was to determine the role of multi detector CT in orbital pathologies, to find the characteristics of CT in orbital pathologies and to correlate the CT findings with histopathological findings wherever possible

Material and methods: The study was conducted in Department of Radiology and Imaging Sciences, Chettinad Hospital and Research Institute, Chennai. Patients with complaints related to traumatic and various non traumatic pathologies were included in our study. All CT examinations were performed using helical CT and reconstructed in appropriate planes

Results: Among 102 patients included in our study, trauma constituted the most common etiology, followed by inflammatory and neoplastic. Lesions were analyzed for calcification, bony and paranasal sinus involvement, enhancement characteristics, lacrimal gland and optic nerve involvement.

Conclusion: CT gives excellent details about bony anatomy in fractures and helps assessing presence or absence of foreign bodies. CT describes the extent of lesions in inflammatory and neoplastic etiologies, accurately assess bony destruction in neoplasms helping in the management of the orbital lesions

Key words: Computed Tomography, Orbital Pathology, Clinicopathological Correlation

\section{INTRODUCTION}

Understanding the imaging findings of common emergent non-traumatic orbital conditions such as inflammation, infection, retinal and choroidal detachment is crucial for their accurate diagnosis and optimal treatment, to avoid permanent visual loss and other potentially devastating consequences. ${ }^{1}$ It is difficult to perform a physical examination on a severely injured patient. Radiography has a sensitivity of $64 \%-78 \%$ for a fracture, but it has very low sensitivity for soft-tissue injuries to the orbital contents. ${ }^{2} \mathrm{CT}$ has been shown to be more accurate than radiography in detecting fractures. When fractures are present, three-dimensional reformation is a useful tool to guide treatment. ${ }^{3}$ Abundance of intraorbital fat provides good intrinsic soft tissue contrast on CT. CT is also valuable in the follow up of tumor regression after surgery, radiotherapy or chemotherapy. ${ }^{4} \mathrm{CT}$ guided fine needle aspiration biopsy has been used in various orbital lesions. ${ }^{5}$ Other applications for orbital CT include ocular motility studies and lacrimal drainage apparatus imaging. ${ }^{6}$ The advances of MDCT technology now make high resolution CT imaging possible.

Study aimed to evaluate the role of computed tomography in orbital pathology, to determine the CT characteristics of different orbital pathology and to correlate CT findings with clinical and histo-pathological findings wherever possible.

\section{MATERIAL AND METHODS}

The study was conducted in Department of Radiology and Imaging Sciences, Chettinad Hospital and Research Institute, Chennai. Patients clinically suspected of having trauma to the orbits, proptosis, pain, visual impairment with or without ophthalmoplegia, orbital mass were subjected ophthalmological examination and CT orbit. CT findings were correlated with final diagnosis based on clinical, laboratory, operative findings, histopathological study or response to treatment.

All CT examinations were performed using Philips Ingenuity Core 128 helical CT. Thin sections $(<1 \mathrm{~mm})$ were acquired in axial plane. Imaging was reformatted in coronal and sagittal planes. Intravenous contrast was used wherever necessary. When orbital varix was suspected, CT was done with and without valsalva maneuver. Clearance was obtained from the ethical committee and informed consent from the patients. Statistical analyses were made with IBM SPSS software version 20.0 and Chi-Square test was applied to determine the statistical significance.

\section{Inclusion criteria}

Patients undergoing CT of brain and orbit in whom there 
were positive findings in orbit.

Patients with history of injury in whom there is orbital finding.

\section{Exclusion criteria}

Patients in whom CT is contraindicated.

\section{RESULTS}

102 patients were included in the study. The distribution of lesions is given in Table 1.

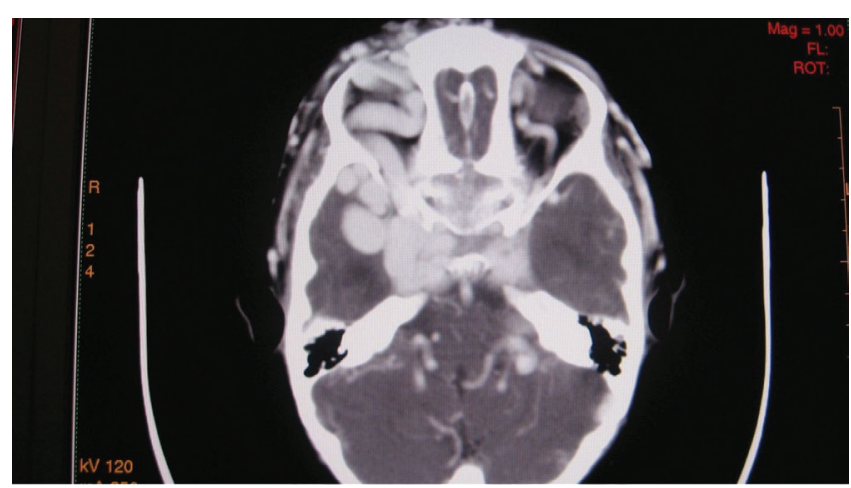

Figure-1: CT angiogram reveals carotico cavernous fistula on right side with dilated superior ophthalmic vein

\begin{tabular}{|l|l|}
\hline Lesion & No. of patients (\%) \\
\hline Congenital & $4(3.92 \%)$ \\
\hline Vascular & $10(9.8 \%)$ \\
\hline Traumatic & $38(37.25 \%)$ \\
\hline Inflammatory & $29(28.43 \%)$ \\
\hline Neoplastic & $21(21.57 \%)$ \\
\hline \multicolumn{2}{|c|}{ Tabe-1: Distributions of various lesions } \\
\hline
\end{tabular}

Among 4 congenital lesions, 1 was coloboma, 2 were dermoid and 1 was cephalocele. Among 10 vascular lesions of the orbit, four were venolymphatic malformation, 2 each

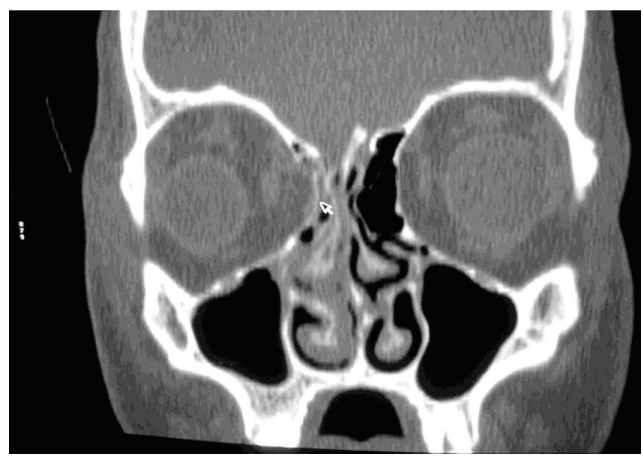

Figure-2: Coronal sections of plain CT of orbits showing blow out fracture of medial wall of right orbit

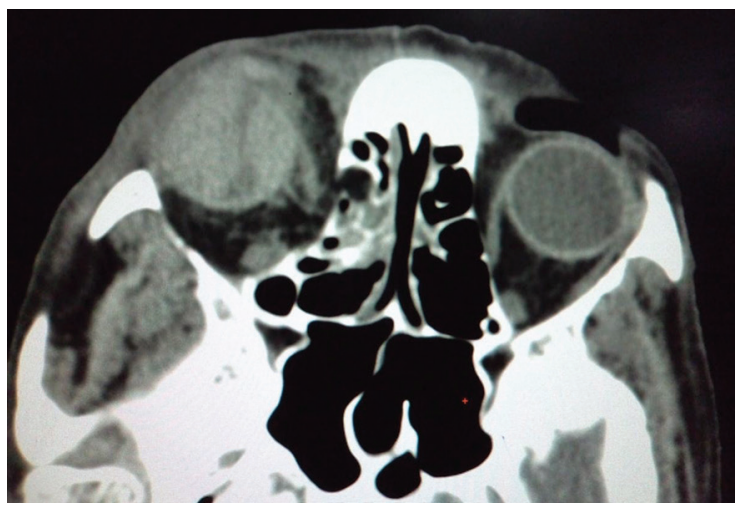

Figure-3: Plain axial section CT showing lenticular shaped choroid hemorrhage in right orbit. Lens is dislocated and is seen anterior to orbit

\begin{tabular}{|l|c|c|c|c|c|c|}
\hline Inflammatory lesions & PNS & $\begin{array}{c}\text { Periorbital } \\
\text { soft tissue }\end{array}$ & EOM & $\begin{array}{c}\text { Intraocular } \\
\text { layers }\end{array}$ & $\begin{array}{c}\text { Lacrimal } \\
\text { gland }\end{array}$ & Optic N. \\
\hline Cellulitis (9) & $6+$ & $9+$ & $3+$ & - & - & $1+$ \\
\hline Orbital abscess (3) & $3+$ & $3+$ & $1+$ & - & - & - \\
\hline Thyroid ophthalmopathy (6) & - & $3+$ & $6+$ & - & - & $1+$ \\
\hline Pseudotumor(3) & - & $3+$ & $3+$ & - & - & - \\
\hline Endophthalmitis.(3) & - & - & - & $3+$ & - & - \\
\hline Panophthalmitis.(3) & - & $3+$ & - & $3+$ & - & $2+$ \\
\hline Dacroadenitis(2) & - & $2+$ & - & - & $2+$ & - \\
\hline & \multicolumn{7}{|l|}{ Table-2: Characterisation of Inflammatory lesions } \\
\hline
\end{tabular}

\begin{tabular}{|l|c|c|c|c|}
\hline Neoplasm & Enhancement & Calcification & Optic $\mathbf{n}$. involvement & Bone destruction \\
\hline Glioma (3) & + & - & + & - \\
\hline Meningioma (4) & + & +3 & + & - \\
\hline Retinoblastoma (5) & + & + & +3 & - \\
\hline Rhabdomyosarcoma (2) & + & - & - & - \\
\hline Melanoma (1) & + & - & - & + \\
\hline Breast metastasis (1) & + & + & - & + \\
\hline NB metastasis (1) & + & - & + & + \\
\hline Ca maxilla (1) & + & - & - & + \\
\hline Ethmoid ca (1) & + & - & - \\
\hline Lacrimal NHL (1) & + & - & - \\
\hline Lacrimal ca (1) & + & & - \\
\hline \multicolumn{7}{|r|}{ Table-3: Characterisation of neoplastic lesions } \\
\hline
\end{tabular}




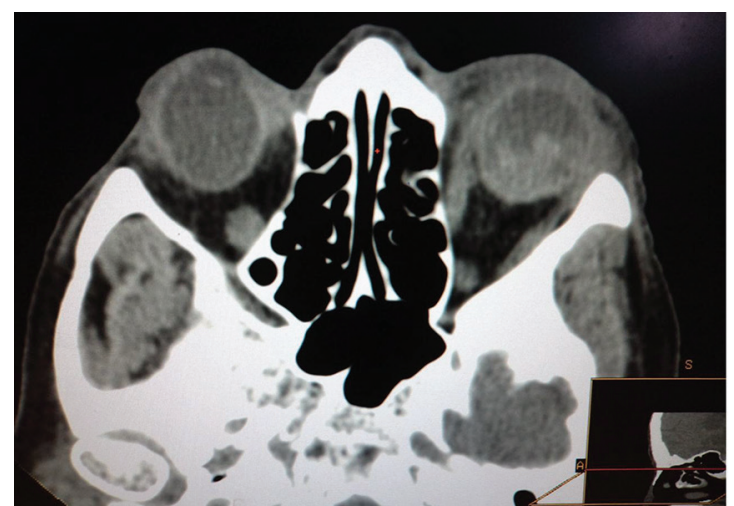

Figure-4: Axial CT section showing vitreous hemorrhage with posterior dislocation of lens

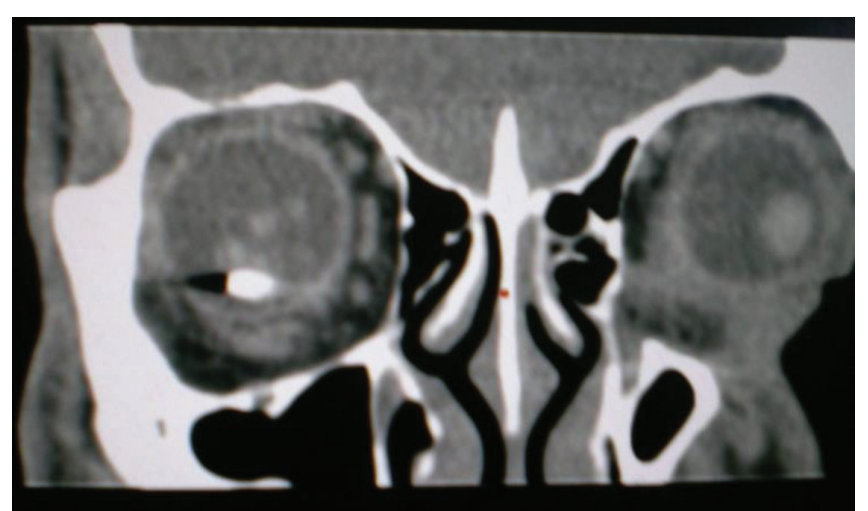

Figure-5: Plain CT coronal sections showing metallic foreign body in the posterior segment of right eyeball

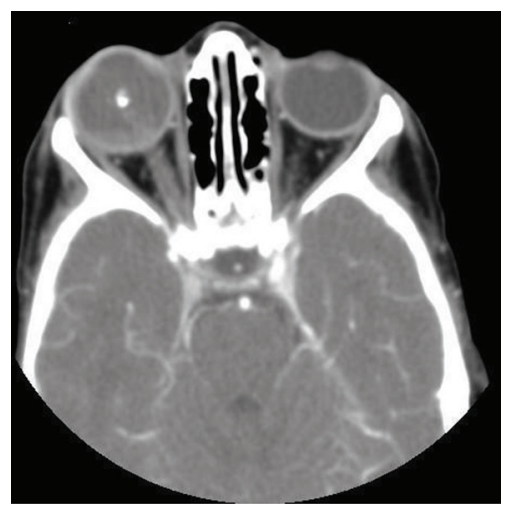

Figure-6: Contrast enhanced CT of retinoblastoma showing enhancing soft tissue mass with calcifications in right orbit

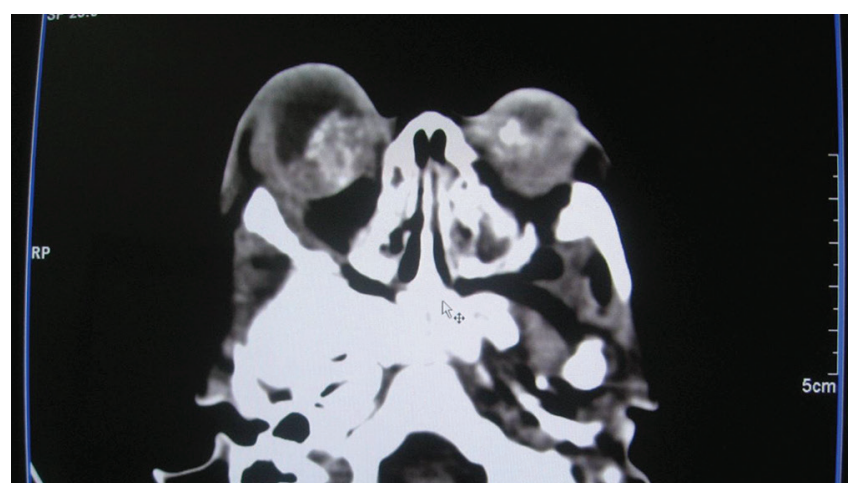

Figure-7: Contrast enhanced CT of bilateral retinoblastoma showing enhancing soft tissue mass with calcifications in bilateral orbits cavernous haemangioma and AV malformation and one each of post traumatic carotico cavernous fistula and orbital varix. One patient with pulsatile exophthalmos and bruit revealed large arteriovenous malformation involving right ophthalmic artery, middle cerebral artery, posterior communicating artery which was confirmed by CT angiography (Figure 1). The extent of involvement in trauma cases as detected by CT were: 38 soft tissue injury, 23 vitreous hemorrhage, 15 foreign body, 14 orbital wall fracture (Figure 2), 9 choroidal detachment (Figure 3), 8 lens dislocation (Figure 4), 8 globe rupture and 2 optic nerve injury. The localization of foreign body: 8 in posterior chamber (Figure 5), 4 in retro orbital compartment and 3 in anterior chamber.

The distribution of inflammatory lesions were as follows: 9 orbital cellulitis, 6 thyroid ophthalmopathy, 3 each of panophthalmitis, endophthalmitis and orbital abscess, 2 dacroadenitis. The characterization of these lesions is depicted in Table 2.

The distribution of neoplasmswere as follows: 5 retinoblastoma (Figures 6, 7), 4 optic nerve sheath meningioma, 3 optic nerve glioma, 2 rhabdomyosarcoma and 1 each of maxillary carcinoma, ethmoidal mass, choroidal melanoma, orbital metastasis from breast carcinoma and neuroblastoma, bilateral lacrimal gland lymphoma and lacrimal carcinoma. CT characterization of these lesions are tabulated in Table 3.

\section{DISCUSSION}

Congenital: In coloboma, NECT shows fluid in defect +/retrobulbar cyst isodense to vitreous. MR or CT both show globe or extraocular features if the defect obscures direct visualization. ${ }^{7}$ It is primarily an ophthalmoscopic diagnosis. Imaging confirms ocular features and evaluates coexistent anomalies. Dermoids appear as well defined non enhancing low density lesions on CT. The posterior extent of complex lesions may not be clinically apparent, therefore imaging is warranted. Irregular scalloping of adjacent bone is a highly suggestive feature. Other bone changes, such as linear defects, thinning, or sclerosis, also occur. ${ }^{8}$ Though MR imaging is the study of choice to illustrate cephaloceles, CT scanning in the sagittal and coronal plane identifies the bony defect as well as the herniated portion of the brain. ${ }^{7}$

Vascular lesions: Capillary hemangioma has irregular margins with prominent enhancement and may be difficult to distinguish from rhabdomyosarcoma. ${ }^{9}$ Cavernous hemangiomas have smooth margins, homogeneous in density, show uniform enhancement and are easily separated from the optic nerve and extraocular muscles. ${ }^{10}$ Imaging of orbital varix demonstrates tortuous tubular channels which taper towards the orbital apex and characteristically lack internal septations. Increase in size following valsalva maneuver or jugular compression is considered pathognomonic. Calcified phleboliths are also characteristic. Varices may appear inherently hyperdense on CT and enhance profusely with contrast. ${ }^{11}$

Traumatic lesions: Interruption of bone continuity, fragments and displacement of bone fragments are direct signs of fracture. Teardrop appearance in the maxillary sinus is a specific indirect sign of inferior orbital fracture. ${ }^{12}$ In cases 
of choroidal detachment, the detachment crosses the optic nerve which distinguishes it from retinal detachment which does not cross ora serrata as retinal epithelium ends at the ora serrata. Retinal detachment with haemorrhage is seen mostly in adults with diabetes mellitus and hypertension in the absence of trauma. ${ }^{13}$ Rarely, a blunt orbital injury may fracture the optic canal and lacerate the optic nerve. More commonly, a definitive fracture is not found. In these cases, the optic nerve or its vascular supply is torn, thrombosed, or compressed. In patients with a rapid posttraumatic decrease in visual acuity, high-resolution $\mathrm{CT}$ of the orbital apex is needed to evaluate for possible fracture. ${ }^{14}$ Evaluation for nonmetallic foreign bodies is more problematic. In a study comparing CT, US, and MR imaging for the ability to demonstrate intraocular glass, $\mathrm{CT}$ was shown to be the most sensitive. Glass fragments of $1.5-\mathrm{mm}$ diameter were detected $96 \%$ of the time and $0.5-\mathrm{mm}$ fragments were detected $48 \%$ of the time. ${ }^{15}$ Unfortunately, glass foreign bodies can still be missed. Unlike metallic and glass foreign bodies, wooden foreign bodies usually appear hypoattenuating on CT; because of low attenuation, they can be mistaken for air. ${ }^{16}$ Wood or organic foreign body should be suspected if the low-attenuation collection seen on CT displays geometric margin. The attenuation of wood can also change over time as their water content changes. Substantial increases in attenuation have been seen in experimental models after $1-5$ days. ${ }^{17}$

Inflammatory lesions: Orbital cellulitis may be associated with serious complications. Prompt diagnosis and proper management are essential for curing the patient. ${ }^{18} \mathrm{CT}$ is a very informative method of evaluating orbital infection. It is capable of demonstrating the presence, location and extent of subperiosteal abscess, intra orbital foreign bodies as well as cerebral complications like cerebritis, brain abscess and epidural infection. ${ }^{19} \mathrm{CT}$ provides tridimensional assessment of the size of the abscess formed as a complication of sinusitis, as well as a clear relation with the adjacent structures. In children, it is critical to distinguish between orbital cellulitis and subperiosteal abscess. The correlation between radiologic and surgical findings, although not absolute, points to CT scan as the paramount means of diagnosis in these cases. ${ }^{20}$ In the study by Nugent et al., the mean extraocular muscle diameters and the calculated muscle diameter index were significantly increased in all orbits with ophthalmopathy, particularly in those with optic neuropathy. Solitary muscle involvement most frequently involved the superior muscle group (6.3\%). ${ }^{21}$ Sagittal imaging with CT or MRI is useful to visualize the entire length of the superior and inferior recti. ${ }^{22}$ Intracranial fat prolapse in combination with optic nerve crowding correlates closely to the presence of optic neuropathy in Graves ophthalmopathy. ${ }^{23}$ Obstruction or invasion of the drainage apparatus by tumor, infection, or posttraumatic scarring is readily depicted by CT. Sagittal/ coronal images reformatted from thin transverse axial sections are often useful in defining the origin of an inferomedial orbital mass and its relation to the lacrimal sac when clinical studies and axial CT findings are equivocal. ${ }^{24}$

Neoplastic lesions: In gliomas, MR is preferred to define proximal optic pathway and to assess related intracranial findings in NF1 patients. But CT is adequate in assessing orbit and sphenoid wing dysplasia. Optic nerve gliomas have a fusiform appearance on CT and MRI, associated with enlargement of the nerve, and have a downward kink in the mid-orbit. There is usually an enlarged optic canal on the side of the lesion, although it is not always associated with intracranial extension of the tumour as arachnoid hyperplasia may also appear similarly. ${ }^{25}$ Optic nerve meningiomas show tram track sign which is most evident on contrast enhanced computed tomography. At CT, osseous changes can be seen in the region of optic canal, such as bone erosion and occasionally hyperostosis. The exact knowledge of bony involvement in meningiomas is imperative because unresected meningiomatosis bone is one of the important factors that may contribute to recurrence. ${ }^{26} \mathrm{In}$ retinoblastoma, the most common intraocular tumor, $\mathrm{CT}$ shows characteristic focal calcification and MRI allows to differentiate it from pseudoglioma. ${ }^{27}$ Punctate/finely speckled calcification is seen in $90-95 \%$ cases on $\mathrm{CT} .{ }^{28}$ In rhabdomyosarcoma, CT shows a mass similar in density to muscle with irregular margins mostly in the extraconal space though half have intraconal extension. These are aggressive tumours with bone erosion and intracranial extension. On MRI, these are usually hypointense to muscle on T1, hyperintense on T2 and show relatively uniform enhancement. ${ }^{29}$ Vast majority of orbital rhabdomyosarcomas are of embryonal sub type. It is important to report the location of the tumour epicenter as there is correlation between location and histology: embryonal sub type more frequently arises in the superior orbit, whereas alveolar sub type is more common in the inferior orbit. ${ }^{30} \mathrm{In}$ melanomas, size of the lesion is a major criteria for therapeutic decision. CT scan provides a better view of the extension into extraorbital region. ${ }^{31}$ Ocular metastasis preferentially affects the vascular-rich choroid membrane. Differential diagnoses include choroidal hemangioma, choroidal nevus and primary intraocular lymphoma. ${ }^{32}$

\section{CONCLUSION}

$\mathrm{CT}$ is excellent in identifying orbital fracture and metallic foreign body, also helps to differentiate orbital cellulitis from pseudotumor and identify Graves disease. CT scan can be considered as a cost effective, noninvasive, reliable diagnostic tool for evaluation of proptosis. ${ }^{33}$ The best protocol is to obtain thin-section axial CT scans, then to perform multiplanar reformation. CT is better for evaluation of bones and detection of calcifications in orbital tumors. CT allows to differentiate rhabdomyosarcoma from dermoid, identification of cavernous haemangioma and many other lesions. $^{28}$

Hence, MDCT is the first investigation in emergency setting with orbital trauma where even MRI is contraindicated in suspected metallic intraocular foreign body. MDCT with its multiplanar reformation techniques also delineates other ocular pathologies with high resolution. MDCT provides essential information for management planning in inflammatory and neoplastic lesions of the orbit.

\section{REFERENCES}

1. Christina A. LeBedis and Osamu Sakai. Nontraumatic 
Orbital Conditions: Diagnosis with CT and MR Imaging in the Emergent Setting. RadioGraphics 2008;28(6):1741-1753.

2. Iinuma T, Hirota $\mathrm{Y}$, Ishio K. Orbital wall fractures: conventional views and CT. Rhinology 1994;32(2): 81-83.

3. Rhea JT, Rao PM, Novelline RA. Helical CT and threedimensional CT of facial and orbital injury. Radiol Clin North Am 1999;37(1):489-513.

4. Vade A, Armstrong D. Orbital Rhabdomyosarcoma in Childhood. Radiol Clin North Am 1987;25(4):701714.

5. Kennerdell, JS, Dubois, PJ, Dekker, A, et al. CT-guided fine needle aspiration biopsy of orbital optic nerve tumors. Ophthalmology 1980; 87(5): 491- 496.

6. Rassell EJ, Huckman M, et al. CT of the inferomedial orbit and the Lacrimal drainage apparatus, Normal and pathologic anatomy. AJNR 1995;6(2):759;766.

7. Vashisht S, Ghai S, Hatimota P, Ghai S, Betharia SM. Cystic lesions of the orbit: A CT spectrum. Indian J Radiol Imaging 2003;13 (2):139-44.

8. Nugent RA, Lapointe JS, Rootman J, Robertson WD, Graeb DA. Orbital dermoids: features on CT. Radiology 1987;165(1):475-478.

9. Bilaniuk LT. Orbital vascular lesions. Role of imaging. Radiol Clin North Am. 1999; 37(1):169-183.

10. Gorospe L, Royo A, Berrocal T, Garcia-Raya P, Moreno $\mathrm{P}$, Abelairas J. Imaging of orbital disorders in paediatric patients. Eur Radiol. 2003;13(5):2012-2026.

11. Orbital imaging: Part 2. Intraorbital pathology. Aviv RI, Miszkiel K. Clin Radiol. 2005;60(3):288-307.

12. Wang Zhenchang, Yan Fei, Tian Qichang, et al. CT Findings of 423 Orbital Fractures, Chinese Journal of Radiology $1995-02$.

13. Poon A,McCluskey PJ,Hill DA. Eye injuries in patients with major trauma. J Trauma 1999;46(2): 494-499.

14. Novelline RA, Liebig T, Jordan J, et al. Computed tomography of ocular trauma. Emerg Radiol 1994;1(3):56-67

15. Kirsch CF, Leen J, et al. Radiologic differentiation of intraocular glass: evaluation of imaging techniques, glass types, size, and effect of intraocular hemorrhage. AJR Am J Roentgenol 2001;177(4):1199-1203

16. Adesanya OO, Dawkins DM. Intraorbital wooden foreign body: mimicking air on CT. Emerg Radiol;14(7):45-49.

17. Yamashita K, Noguchi T, Mihara F, et al. An intraorbital wooden foreign body: description of a case and a variety of CT appearances. Emerg Radiol 2007; 14(5):41-43.

18. Botting AM, MCIntosh, Mahadevan M. Pediatric pre and post-septal infections are different diseases. A Retrospective Review of 262 cases. Int J Otorhinolaryngology 2008;72(4):377.

19. Robert A Zimmerman, Larissa $\mathrm{T}$ Bilaniuk. CT Of Orbital Infection and its Cerebral Complications. Am J Radiol 2000;134(6):45-50.

20. Ognibene RZ, Voegels RL, Bensodon RL, Butugan. Orbital Complications of sinusitis. Am J Rhino 2004;8(4):175-9.

21. R A Nugent, R I Belkin, J M Neigel, J Rootman, et al. Graves orbitopathy: Correlation of CT and clinical findings. Radiology, 1990;177(6):675-682.

22. Kahaly GJ: Imaging in thyroid-associated orbitopathy. Eur J Endocrinol 2001;145(2): 107-18.

23. D Birchall, K L Goodall, J L Noble, A Jackson. Graves ophthalmopathy: intracranial fat prolapse on CT images as an indicator of optic nerve compression. Radiology, 1996;200(4):123-127.

24. Russell EJ, Czervionke L, Huckman M. CT of the inferomedial orbit and the lacrimal drainage apparatus: normal and pathologic anatomy. AJR Am J Roentgenol. 1995;145(6):1147-54.

25. Shapey J, Danesh-Meyer HV, Kaye AH. Review: Diagnosis and management of optic nerve glioma. Journal Of Clinical Neuroscience. 2011;18(5):15851591.

26. Uday S. Kanamalla. The Optic Nerve - Tram-Track Sign. Radiology, 2003; 227(3):718-719.

27. Hopper KD, Sherman JL, Boal DK, Eggli KD. CT and MR Imaging Of the Pediatric Orbit. Radiographics, 1992;12(3): 485-503.

28. Tejas Kalyanpur, Mathew Cheriyan. Role of MRI and CT in Ocular and Orbital Diseases. Kerala Journal of Ophthalmology. December 2009;21(4):430-441.

29. Gorospe L, Royo A, Berrocal T, Garcia-Raya P, Moreno $\mathrm{P}$, Abelairas J. Imaging of orbital disorders in paediatric patients. Eur Radiol. 2003; 13(6):2012-2026

30. Mccarville MB, Spunt SL, Pappo AS. Rhabdomyosarcoma in pediatric patients: the good, the bad and the unusual. AJR Am J Roentgenol. 2001;176 (6): 1563-9.

31. A.Kharat, A.Singh, N.Naphade. Imaging Finding In A Case of Recurrent Malignant Melanoma Of Eyelid. Indian Journal Of Radiology Imaging 2005;15(1):4951.

32. Mysore N, Gonçalves F, Chankowsky J, del CarpioO'Donovan R. Adult orbital masses: a pictorial review. Canadian Association Of Radiologists Journal February 2012;63(1):39-46.

33. KK Sabharwal, AL Chouhan, S Jain. CT Evaluation Of Proptosis. Ind J Radiology Imaging 2006;16(4):683688.

\section{Source of Support: Nil; Conflict of Interest: None}

Submitted: 05-04-2018; Accepted: 03-05-2018; Published online: 10-05-2018 